\title{
A novel inhibitor of Rho GDP-dissociation inhibitor $\alpha$ improves the therapeutic efficacy of paclitaxel in Lewis lung carcinoma
}

\author{
XING CHEN PENG ${ }^{1 *}$, XU XIA CHEN ${ }^{1 *}$, YU ZHANG ${ }^{2 *}$, HAI JUN WANG ${ }^{3 *}$ and YOU FENG ${ }^{1}$ \\ ${ }^{1}$ Department of Medical Oncology, Cancer Center, State Key Laboratory of Biotherapy, West China Hospital, \\ Sichuan University, Chengdu, Sichuan 610041; ${ }^{2}$ Department of Oncology, Guizhou Provincial People's Hospital, Guiyang, \\ Guizhou 550000, ${ }^{3}$ Department of Thoracic Surgery, Sichuan Cancer Hospital, Chengdu, Sichuan 610041, P.R. China
}

Received April 5, 2015; Accepted May 21, 2015

DOI: $10.3892 /$ br.2015.475

\begin{abstract}
Molecular-targeted therapies are considered a promising strategy for the treatment of most types of human cancer. Rho GDP-dissociation inhibitor $\alpha$ (RhoGDI $\alpha$ ), which functions mainly by controlling the cellular distribution and activity of Rho GTPases and is associated with tumor progression and poor prognosis of cancer patients, has become a new promising target for anticancer treatment. Recently, a specific RhoGDI $\alpha$ inhibitor (no. SKLB-163) was developed via computer-aided drug design and de novo synthesis. Previous studies have shown that SKLB-163 had extremely good antitumor activities against diverse cancer cell lines. In the present study, SKLB-163 was used in combination with paclitaxel in order to determine the synergistic effect of the antitumor activity. The findings showed that the combination therapy clearly inhibited cell proliferation and induced apoptosis of LL/2 in vitro. The LL/2 mice model also showed that the combination therapy inhibited tumor growth in vivo. Proliferative cell nuclear antigen (PCNA) immunohistochmeistry and terminal deoxynucleotidyl transferase dUTP nick end-labeling showed that combination therapy inhibited cell proliferation and increased apoptosis compared to the treatment with SKLB-163 or paclitaxel alone. The data suggests that the combination therapy exerted synergistic antitumor effects, providing a novel way to augment the antitumor efficacy of cytotoxic chemotherapy.
\end{abstract}

Correspondence to: Dr Xing Chen Peng, Department of Medical Oncology, Cancer Center, State Key Laboratory of Biotherapy, West China Hospital, Sichuan University, 37 GuoXue Xiang, Chengdu, Sichuan 610041, P.R. China

E-mail: pxx2014@sohu.com

${ }^{*}$ Contributed equally

Key words: Rho GDP-dissociation inhibitor $\alpha$, molecular-targeted therapy, paclitaxel, proliferation, apoptosis

\section{Introduction}

Cancer cells rely on critical signaling pathways for their proliferation, invasion and metastasis. Critical signaling molecules, which function particularly at points where several of these pathways crosstalk, provide valuable targets for the development of novel anticancer drugs (1).

Rho GDP-dissociation inhibitor $\alpha$ (RhoGDI $\alpha)$, which is a member of a family of GDIs that include D4-GDI, RhoGDI-3 and RhoGDI $\alpha$, functions mainly by affecting the cellular distribution and activity of Rho GTPases (2-4). RhoGDI $\alpha$ can negatively modulate Rho proteins by three methods: i) By suspending their interaction with guanine nucleotide exchange factors, thereby inhibiting GTPase activation; ii) by shielding the membrane-anchoring domain of the GTPases, thereby restricting them to a cytosolic localization; and iii) by blocking the binding to downstream target molecules (5). The expression of RhoGDI $\alpha$ is upregulated in diverse types of human cancer, including lung cancer, breast cancer and melanoma $(6,7)$. Overexpression of RhoGDI $\alpha$ is associated with tumor progression and poor prognosis (8). The critical role of RhoGDI $\alpha$ in cancer cell function and their role in cancer etiology highlights the importance of RhoGDI $\alpha$ as a novel target for anticancer treatment.

In our previous study, a specific RhoGDI $\alpha$ inhibitor (no. SKLB-163) was developed via computer-aided drug design and de novo synthesis in our laboratory. Our data exhibited that SKLB-163 had good anticancer activities in vitro and in vivo $(9,10)$. The molecular mechanism is involved as follows: SKLB-163 inhibited the upstream RhoGDI $\alpha$ protein and activated that c-Jun $\mathrm{N}$-terminal kinase 1 signaling pathway that could contribute to the activation of caspase-3, decreased level of phosphorylated mitogen-activated protein kinase and AKT. To fully explore the potential of SKLB-163, the antitumor efficacy of the combination of SKLB-163 and paclitaxel was evaluated in the LL/2 mice model in the present study. The findings showed that the combination therapy clearly inhibited cell proliferation and induced apoptosis of LL/2 in vitro. The LL/2 mice model also showed that the combination therapy inhibited tumor growth in vivo. Proliferative cell nuclear antigen (PCNA) immunohistochemistry and terminal deoxynucleotidyl transferase dUTP nick end-labeling (TUNEL) showed that combination therapy inhibited cell proliferation 
and increased apoptosis compared to the treatment with SKLB-163 or paclitaxel alone. The data suggests that the combination therapy exerted synergistic antitumor effects, providing a novel way to augment the antitumor efficacy of cytotoxic chemotherapy.

\section{Materials and methods}

Synthesis of SKLB-163. The route adapted for the synthesis of the SKLB-163 compound was performed as previously described (10). SKLB-163 was dissolved in dimethyl sulfoxide (DMSO) as a stock solution and was stored at $4^{\circ} \mathrm{C}$. In the in vitro study, the stock solution was diluted in cell culture medium at a final DMSO concentration of $0.05 \%(\mathrm{v} / \mathrm{v})$. The formulation used in the in vivo study contains SKLB-163, $0.5 \%$ carboxymethylcellulose and $1 \%$ glycerin.

Cell culture. The LL/2 murine Lewis lung cancer cell line, CT26 murine colon adenocarcinoma cell line, B16 murine melanoma cell line, HB1 human bronchial epithelial cell line, LO2 human liver cell line and HEK293 human embryonic kidney cell line were grown in RPMI-1640 (Invitrogen Life Technologies, Bedford, MA, USA) or Dulbecco's modified Eagle's medium (Invitrogen Life Technologies) containing $10 \%$ heat-inactivated fetal bovine serum, $100 \mathrm{U} / \mathrm{ml}$ penicillin and $100 \mathrm{U} / \mathrm{ml}$ streptomycin in a humidity chamber at $37^{\circ} \mathrm{C}$ under a $5 \% \mathrm{CO}_{2}$ in atmosphere.

Cell viability assay. Cells were seeded at $4-5 \times 10^{3}$ cells/well in 96-well plates and were allowed to attach overnight at $37^{\circ} \mathrm{C}$. Subsequently, medium containing agents were added to each well and cells were further cultured at $37^{\circ} \mathrm{C}$ for $48 \mathrm{~h}$. Cell viability was estimated using the MTT assay. The absorbance was measured at $570 \mathrm{~nm}$ with a microplate reader (Bio-Rad, Berkeley, CA, USA).

Quantitative assessment of apoptosis. Apoptotic cells treated with corresponding agents were further analyzed by a flow cytometer. Collected cells were stained with $1 \mathrm{ml}$ hypotonic fluorochrome solution containing $50 \mu \mathrm{g} / \mathrm{ml}$ propidium iodide in $0.1 \%$ sodium citrate plus $0.1 \%$ Triton X-100. Subsequently, flow cytometric analysis was performed to identify apoptotic cells or sub- $\mathrm{G}_{1}$ cells to measure the percentage of sub- $\mathrm{G}_{1}$ cells (ESP Elite; Beckman Coulter, Brea, CA, USA). Apoptotic cells had less DNA content than that of the $G_{1}$ cells in the cell cycle distribution and the results were estimated with list mode software.

In vivo tumor experiment. The study received approval from the Ethics Committee of Sichuan University (Sichuan, China). The animal studies were approved by the Institutional Animal Care and Treatment Committee of Sichuan University. C57BL/6 mice, 6 to 8 weeks old, were obtained from the Experimental Animal Center of Sichuan University and were housed in our animal research facility. LL/ 2 cells $\left(\sim 1 \times 10^{6}\right)$ in $0.1 \mathrm{ml}$ of phosphate-buffered saline (PBS) were injected subcutaneously into the right oxter of each mouse. When the diameter of the tumors reached up to 6-8 $\mathrm{mm}$, animals were randomized into one of the following 4 groups: Control, normal saline-treated group; SKLB-163 group, $100 \mathrm{mg} / \mathrm{kg}$ by intragastric administration
Table I. Cytotoxicity effect of SKLB-163

\begin{tabular}{llc}
$\begin{array}{l}\text { Tumor } \\
\text { cell line }\end{array}$ & \multicolumn{1}{c}{ Cell type } & $\mathrm{IC}_{50}, \mu \mathrm{mol} / \mathrm{l}$ \\
\hline LL/2 & Murine Lewis lung cancer & $2.45 \pm 0.67$ \\
CT26 & Murine colon adenocarcinoma & $7.56 \pm 1.34$ \\
B16 & Murine melanoma & $6.31 \pm 1.27$ \\
HB1 & Human bronchial epithelial & $>40$ \\
LO2 & Human liver & $>40$ \\
HEK293 & Human embryonic kidney & $>40$
\end{tabular}

Each cell line was treated with various concentrations $(0-20 \mu \mathrm{mol} / \mathrm{l})$ of SKLB-163 for $48 \mathrm{~h}$, respectively. Cell viability was detected by the MTT assay. Data are expressed as the mean \pm standard deviation (SD) from three experiments.

once daily; paclitaxel group, $10 \mathrm{mg} / \mathrm{kg}$ by intraperitoneal (i.p.) injection once a week; and the SKLB-163+paclitaxel group, SKLB-163 (100 mg/kg by intragastric administration once daily) and paclitaxel (10 mg/kg by i.p. injection once a week). Tumor growth was evaluated every 3 days by measurement of tumor diameters and the volume of the tumor was determined using the formula: Volume $\left(\mathrm{mm}^{3}\right)=$ length $\mathrm{x}$ width ${ }^{2} \mathrm{x} 0.52$, as previously described (11). After all the mice from each group were sacrificed, the tumor net weight of each mouse was measured.

PCNA immunohistochemistry. The tumor sections were stained by the EnVision ${ }^{\mathrm{TM}}+$ System-horseradish peroxidase method (DakoCytomation, Carpinteria, CA, USA), according to the manufacturer's instructions. The primary antibody for PCNA was purchased from Santa Cruz Biotechnology, Inc., (Dallas, TX, USA; rabbit anti-mouse; cat. no. sc-7907).

TUNEL assay. The presence of apoptotic cells within the tumor sections was evaluated by the TUNEL technique using the DeadEnd ${ }^{\mathrm{TM}}$ Fluorometric TUNEL System (Promega, Madison, WI, USA) following the manufacturer's instructions. Percent apoptosis was determined by counting the number of apoptotic cells and dividing by the total number of cells in the field (5 high power fields/slide).

\section{Results}

Cytotoxicity effect of SKLB-163. SKLB-163 was synthesized in our laboratory (Fig. 1A). In the present study, 3 murine cancer cell lines (LL/2, CT26 and B16) and normal cell lines (HB1, LO2 and HEK293) were used to investigate the cytotoxicity of SKLB-163. After $48 \mathrm{~h}$ treatment, SKLB-163 inhibited the viability of all the murine cancer cell lines. $\mathrm{The}^{\mathrm{IC}_{50}}$ is shown in Table I. Additionally, no apparent toxicity on normal cells was observed (Table I).

SKLB-163 enhances LL/2 cell sensitivity to paclitaxel in vitro. In order to investigate whether SKLB-163 could affect LL/2 cell sensitivity to paclitaxel (Fig. 1B), the MTT assay and flow cytometry were carried out. Cell viability of LL/2 cells 
A

B<smiles>O=C(CCl)NCc1ccccc1</smiles>

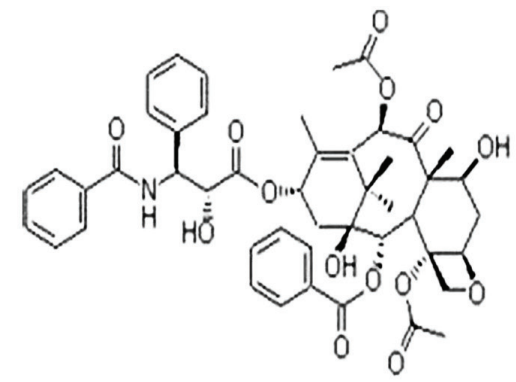

Figure 1. Structure of (A) SKLB-163 and (B) paclitaxel.
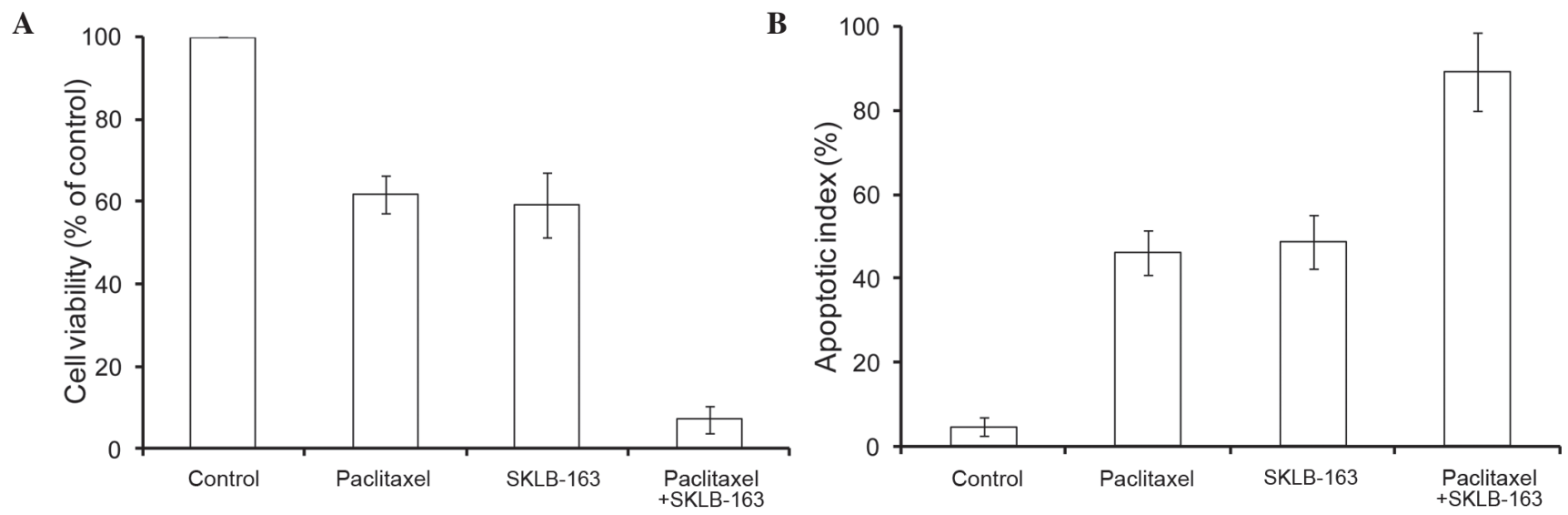

Figure 2. SKLB-163 enhances LL/2 cell sensitivity to paclitaxel in vitro. (A) Cell viability of LL/2 cells was significantly reduced when treated with SKLB-163+paclitaxel compared with paclitaxel alone and SKLB-163 alone $(\mathrm{P}<0.05)$. (B) Apoptosis was markedly enhanced in the SKLB-163+paclitaxel-treated group, compared to treatment with paclitaxel alone, SKLB-163 alone and the phosphate-buffered saline control $(\mathrm{P}<0.05)$.

A

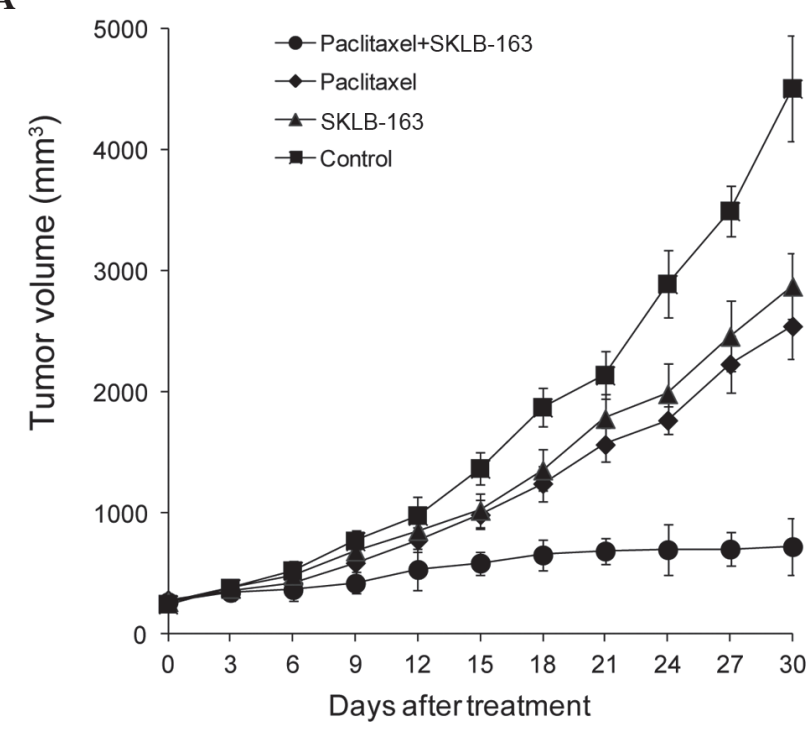

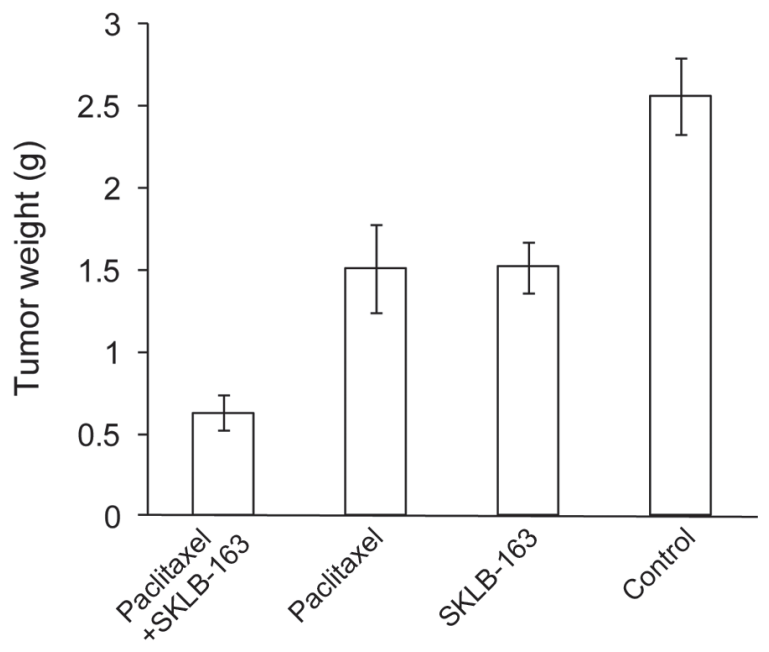

Figure 3. Antitumor effects of SKLB-163+paclitaxel in the LL/2 mice model. (A) Tumor growth was significantly inhibited in the combination therapy treated group compared with the controls (including paclitaxel alone, SKLB-163 alone and normal saline control). (B) Similar results were also found for the tumor weight.

was significantly reduced in the SKLB-163 plus paclitaxel treated group compared to the treatment with paclitaxel alone $(1.5 \mathrm{nM})$, SKLB-163 alone $(2 \mu \mathrm{mol} / \mathrm{l})$ and PBS control (Fig. 2A). In addition, apoptosis was markedly enhanced in the SKLB-163 plus paclitaxel-treated group compared to the treatment with paclitaxel alone (1.5 nM), SKLB-163 alone (2 $\mu \mathrm{mol} / \mathrm{l})$ and PBS control (Fig. 2B).

Antitumor effect of combination therapy in vivo. In order to study the antitumor effect of combination therapy, the LL/2 
A

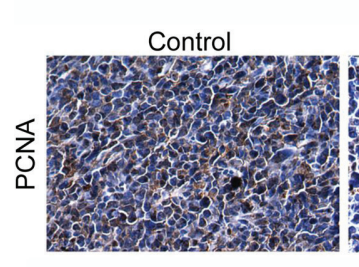

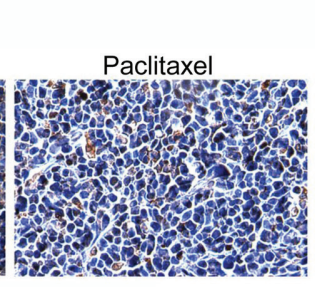

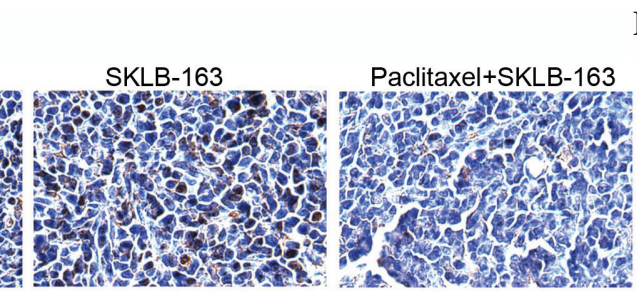

B

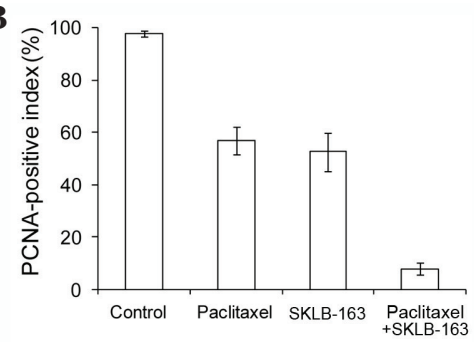

Figure 4. Histological analysis by proliferative cell nuclear antigen (PCNA) immunoreactivity analysis in LL/2 tumor models. (A) Representative images from each group. (B) Quantified values shown are the average percentage of PCNA-positive nuclei.

A

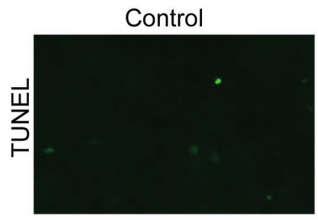

Paclitaxel

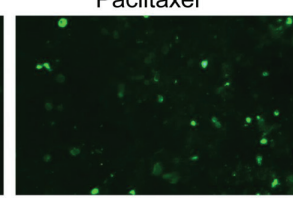

SKLB-163

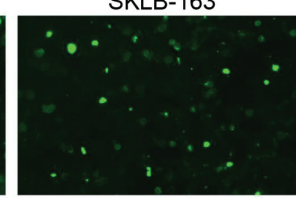

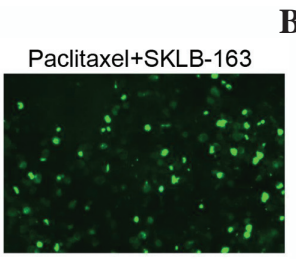

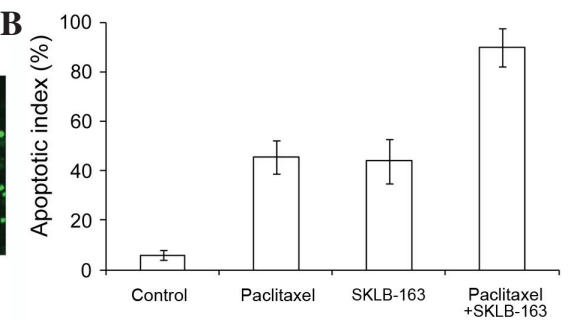

Figure 5. Induction of tumor cell apoptosis estimated by terminal deoxynucleotidyl transferase dUTP nick end-labeling staining. (A) Apoptotic tumor cells were elevated within tumor tissues obtained from the combination therapy group, compared with paclitaxel, SKLB-163 and normal saline mice. (B) The apoptotic index was calculated as a ratio of the apoptotic cell number to the total cell number in each field.

mice model was established. As shown in Fig. 3A, single paclitaxel or SKLB-163 treatment suppressed tumor growth and the tumor inhibition rate was 43 and $36 \%$, respectively, in the LL/2 model. However, the combination therapy significantly decreased the tumor volume and resulted in $84 \%$ tumor regression. Similar results were also found for the tumor weight (Fig. 3B). All the data showed that SKLB-163 increased the suppression of tumor growth induced by paclitaxel.

Combination therapy inhibited proliferation in vivo. To obtain additional insight into the in vivo effects, tumor cell proliferation was assessed by PCNA immunoreactivity analysis. As shown in Fig. 4, combination therapy clearly reduced percentages of PCNA-positive nuclei in LL/2 tumor models when compared with the tumors from the control group.

Combination therapy increased apoptosis in vivo. In the TUNEL assay to evaluate apoptosis in vivo, a significantly greater percentage of TUNEL-positive nuclei could be observed in the combination therapy group when compared with the tumors from the control groups (Fig. 5).

\section{Discussion}

Due to the high degree of cancer clonal heterogeneity and cell signal complexity, downregulation of a single target does not necessarily eradicate the cancer. Therefore, traditional chemotherapy combined with targeted agents may be the most effective way to improve treatment efficacy and overcome resistance in oncotherapy (1).

The present study investigated the synergistic antitumor effects of SKLB-163 used in combination with paclitaxel in the murine LL/2 model. SKLB-163, a new benzothiazole-2-thiol derivative, was developed via a computer-aided drug design and de novo synthesis. SKLB-163 showed significant cytotoxicity against various murine cancer cells by the MTT assay. A clear increased suppression of tumor cell proliferation and increased induction of apoptosis were evidenced in the combination therapy group by the MTT assay and flow cytometry. The in vivo effects were explored in LL/2 mice models. Single paclitaxel or SKLB-163 suppressed tumor growth and the inhibition rate of the tumor was 43 and $36 \%$, respectively, in the LL/2 model. However, the combination therapy significantly decreased the tumor volume and resulted in $84 \%$ tumor regression. To obtain additional insight into the in vivo effects, tumor cell proliferation and apoptosis were assessed by PCNA immunoreactivity analysis and the TUNEL assay. Data exhibited that combination therapy clearly reduced the percentages of PCNA-positive nuclei and increased the percentages of TUNEL-positive nuclei.

Benzothiazole derivatives have been known for diverse biological functions, including antitubercular, antimalarial, antihelmintic, analgesic, anticonvulsant, anti-inflammatory and antitumor activities $(12,13)$. The benzothiazole moiety modified with certain functional groups, such as imidazole and aryl, can significantly inhibit the growth of certain cancer cell lines (14-16). However, the majority of studies focused on designing new benzothiazole compounds by substituting 2-aminobenzothiazoles or 2-arylbenzothiazoles; only a few investigators employed the benzothiazole-2-thiol as a functional group. In the present study, SKLB-163, a new benzothiazole-2-thiol derivative, was developed via computer-aided drug design and de novo synthesis.

SKLB-163 has numerous advantages. Firstly, the synthetic route is easy to handle and cost is low. Secondly, oral administration is usually safe and convenient, which patients can easily accept and adopt. Finally, SKLB-163 has broad-spectrum antitumor activity as RhoGDI is overexpressed in multiple types of human cancer. 
In conclusion, the data suggests that SKLB-163 combined with paclitaxel exerted synergistic antitumor effects, providing a novel way to augment the antitumor efficacy of cytotoxic chemotherapy.

\section{Acknowledgements}

The present study was supported by the National Natural Sciences Foundation of China (grant no. 81402494) and tbe Science and Technology Support Program of Sichuan province (grant nos. 2015SZ0076 and 2014HH0063).

\section{References}

1. Li F, Zhao C and Wang L: Molecular-targeted agents combination therapy for cancer: Developments and potentials. Intl J Cancer 134: 1257-1269, 2014.

2. Olofsson B: Rho guanine dissociation inhibitors: Pivotal molecules in cellular signalling. Cell Signal 11: 545-554, 1999.

3. Sasaki T and Takai Y: The Rho small G protein family-Rho GDI system as a temporal and spatial determinant for cytoskeletal control. Biochem Biophys Res Commun 245: 641-645, 1998.

4. Golovanov AP, Chuang TH, DerMardirossian C, Barsukov I, Hawkins D, Badii R, Bokoch GM, Lian LY and Roberts GC: Structure-activity relationships in flexible protein domains: Regulation of rho GTPases by RhoGDI and D4 GDI. J Mol Biol 305: 121-135, 2001.

5. Zhang B, Zhang Y, Dagher MC and Shacter E: Rho GDP dissociation inhibitor protects cancer cells against drug-induced apoptosis. Cancer Res 65: 6054-6062, 2005.

6. Poland J, Schadendorf D, Lage H, Schnolzer M, Celis JE and Sinha P: Study of therapy resistance in cancer cells with functional proteome analysis. Clin Chem Lab Med 40: 221-234, 2002.

7. Sinha P, Kohl S, Fischer J, Hütter G, Kern M, Köttgen E, Dietel M, Lage H, Schnölzer M and Schadendorf D: Identification of novel proteins associated with the development of chemoresistance in malignant melanoma using two-dimensional electrophoresis Electrophoresis 21: 3048-3057, 2000.
8. Zhao L, Wang H, Li J, Liu Y and Ding Y: Overexpression of Rho GDP-dissociation inhibitor alpha is associated with tumor progression and poor prognosis of colorectal cancer. J Proteome Res 7: 3994-4003, 2008.

9. Peng X, Xie G, Wang Z, Lin H, Zhou T, Xiang P, Jiang Y, Yang S, Wei Y, Yu L, et al: SKLB-163, a new benzothiazole-2-thiol derivative, exhibits potent anticancer activity by affecting RhoGDI/JNK-1 signaling pathway. Cell Death Dis 5: e1143, 2014.

10. Wang Z, Shi XH, Wang J, Zhou T, Xu YZ, Huang TT, Li YF, Zhao YL, Yang L, Yang SY, et al: Synthesis, structure-activity relationships and preliminary antitumor evaluation of benzothiazole-2-thiol derivatives as novel apoptosis inducers. Bioorg Med Chem Lett 21: 1097-1101, 2011.

11. Shi W, Tang Q, Chen X, Cheng P, Jiang P, Jing X, Chen X, Chen P, Wang Y, Wei Y, et al: Antitumor and antimetastatic activities of vesicular stomatitis virus matrix protein in a murine model of breast cancer. J Mol Med Berl 87: 493-506, 2009.

12. Hutchinson I, Chua MS, Browne HL, Trapani V, Bradshaw TD, Westwell AD and Stevens MF: Antitumor benzothiazoles. 14. Synthesis and in vitro biological properties of fluorinated 2-(4-aminophenyl)benzothiazoles. J Med Chem 44: 1446-1455, 2001.

13. Kok SH, Gambari R, Chui CH, Yuen MC, Lin E, Wong RS, Lau FY, Cheng GY, Lam WS and Chan SH: Synthesis and anti-cancer activity of benzothiazole containing phthalimide on human carcinoma cell lines. Bioorg Med Chem 16: 3626-3631, 2008.

14. Trapani G, Franco M, Latrofa A, Reho A and Liso G: Synthesis, in vitro and in vivo cytotoxicity, and prediction of the intestinal absorption of substituted 2-ethoxycarbonyl-imidazo[2,1-b] benzothiazoles. Eur J Pharm Sci 14: 209-216, 2001.

15. Song EY, Kaur N, Park MY, Jin Y, Lee K, Kim G, Lee KY, Yang JS, Shin JH, Nam KY, et al: Synthesis of amide and urea derivatives of benzothiazole as Raf-1 inhibitor. Eur J Med Chem 43: 1519-1524, 2008.

16. Srimanth K, Rao VR and Krishna DR: Synthesis and evaluation of anticancer activity of some imidazothiazolyl, imidazobenzothiazolyl and dihydroimidazothiazolyl coumarins. Arzneimittelforschung 52: 388-392, 2002. 